\title{
On the relationships between the Michaelis-Menten kinetics, reverse Michaelis-Menten kinetics, equilibrium chemistry approximation kinetics, and quadratic kinetics
}

\author{
J. Y. Tang \\ Department of Climate Sciences, Lawrence Berkeley National Laboratory, Berkeley, CA, USA \\ Correspondence to: J. Y. Tang (jinyuntang@lbl.gov) \\ Received: 7 August 2015 - Published in Geosci. Model Dev. Discuss.: 3 September 2015 \\ Revised: 16 November 2015 - Accepted: 18 November 2015 - Published: 1 December 2015
}

\begin{abstract}
The Michaelis-Menten kinetics and the reverse Michaelis-Menten kinetics are two popular mathematical formulations used in many land biogeochemical models to describe how microbes and plants would respond to changes in substrate abundance. However, the criteria of when to use either of the two are often ambiguous. Here I show that these two kinetics are special approximations to the equilibrium chemistry approximation (ECA) kinetics, which is the first-order approximation to the quadratic kinetics that solves the equation of an enzyme-substrate complex exactly for a single-enzyme and single-substrate biogeochemical reaction with the law of mass action and the assumption of a quasi-steady state for the enzyme-substrate complex and that the product genesis from enzyme-substrate complex is much slower than the equilibration between enzymesubstrate complexes, substrates, and enzymes. In particular, I show that the derivation of the Michaelis-Menten kinetics does not consider the mass balance constraint of the substrate, and the reverse Michaelis-Menten kinetics does not consider the mass balance constraint of the enzyme, whereas both of these constraints are taken into account in deriving the equilibrium chemistry approximation kinetics. By benchmarking against predictions from the quadratic kinetics for a wide range of substrate and enzyme concentrations, the Michaelis-Menten kinetics was found to persistently underpredict the normalized sensitivity $\partial \ln v / \partial \ln k_{2}^{+}$ of the reaction velocity $v$ with respect to the maximum product genesis rate $k_{2}^{+}$, persistently overpredict the normalized sensitivity $\partial \ln v / \partial \ln k_{1}^{+}$of $v$ with respect to the intrinsic substrate affinity $k_{1}^{+}$, persistently overpredict the normalized sensitivity $\partial \ln v / \partial \ln [E]_{T}$ of $v$ with respect the to-
\end{abstract}

tal enzyme concentration $[E]_{T}$, and persistently underpredict the normalized sensitivity $\partial \ln v / \partial \ln [S]_{T}$ of $v$ with respect to the total substrate concentration $[S]_{T}$. Meanwhile, the reverse Michaelis-Menten kinetics persistently underpredicts $\partial \ln v / \partial \ln k_{2}^{+}$and $\partial \ln v / \partial \ln [E]_{T}$, and persistently overpredicts $\partial \ln v / \partial \ln k_{1}^{+}$and $\partial \ln v / \partial \ln [S]_{T}$. In contrast, the equilibrium chemistry approximation kinetics always gives consistent predictions of $\partial \ln v / \partial \ln k_{2}^{+}, \partial \ln v / \partial \ln k_{1}^{+}$, $\partial \ln v / \partial \ln [E]_{T}$, and $\partial \ln v / \partial \ln [S]_{T}$, indicating that ECAbased models will be more calibratable if the modeled processes do obey the law of mass action. Since the equilibrium chemistry approximation kinetics includes advantages from both the Michaelis-Menten kinetics and the reverse Michaelis-Menten kinetics and it is applicable for almost the whole range of substrate and enzyme abundances, land biogeochemical modelers therefore no longer need to choose when to use the Michaelis-Menten kinetics or the reverse Michaelis-Menten kinetics. I expect that removing this choice ambiguity will make it easier to formulate more robust and consistent land biogeochemical models.

\section{Introduction}

The recent recognition that the typical turnover-pool-based soil carbon models cannot model the priming effect has revived the interest in developing microbe explicit soil biogeochemistry models. This has been manifested in a long list of microbial models that were published in the last few years (e.g., Schimel and Weintrub, 2003; Moorhead and Sinsabaugh, 2006; Allison et al., 2010; German et al., 2012; 
Wang et al., 2013; Wieder et al., 2013; Li et al., 2014; He et al., 2014; Riley et al., 2014; Xenakis and Williams, 2014; Tang and Riley, 2015; Sulman et al., 2014; Wieder et al., 2015). To build a microbial model, the substrate kinetics is fundamental as it describes the rate at which microbes would take up a substrate and represents the first step towards describing how microbes would decompose the soil organic matter. Under the assumption that a single "master reaction" limits the growth of microbes (Johnson and Lewin, 1946), the substrate kinetics even completely determines the microbial dynamics as done in many models (e.g., the Monod model). Among the many mathematical formulations of substrate kinetics (see Tang and Riley, 2013 for a review), the MichaelisMenten (MM) kinetics is often used because it has succeeded in many applications ever since its creation in the early 20th century (Michaelis and Menten, 1913). However, Schimel and Weintraub (2003) proposed in their study that the decomposition rate should vary more like an asymptotic function of enzyme abundance such that the reverse Michaelis-Menten (RMM) kinetics would better model the soil carbon decomposition dynamics. The proposal of RMM kinetics was motivated by the empirical observation that, as enzyme concentration increases, microbial growth cannot increase continuously without a limit; therefore, some dynamic feedbacks between the different components must stabilize the system. In contrast, the MM kinetics predicts that substrate degradation is proportional to enzyme concentration and, therefore, like the linear kinetics, as used in Schimel and Weintraub (2003), it will predict unstable decomposition dynamics. The success by Schimel and Weintraub has led to a number of studies using the RMM kinetics as the backbone of their microbial models, including the Moorhead and Sinsabaugh (2006) model of litter decomposition, the Drake et al. (2013) model for root priming, the Waring et al. (2013) model for change in microbial community structure in soil carbon and nitrogen cycling, and the Averill (2014) model for change in microbial allocation in soil carbon decomposition.

Wang and Post (2013) pointed out that both the MM kinetics and RMM kinetics (although the latter is empirical) are special approximations to the quadratic kinetics that exactly solves for the enzyme-substrate complex under the quasi-steady-state approximation (QSSA), which states that the enzyme-substrate complexes are in instantaneous equilibrium with enzyme and substrate concentrations (Borghans et al., 1996). They further concluded that the MM kinetics is applicable when the substrate concentration far exceeds the enzyme concentration, and the RMM kinetics is applicable when either the enzyme concentration far exceeds the substrate concentration or vice versa. The condition for the MM kinetics to be applicable as provided by Wang and Post (2013) was however much narrower than that was proposed in some earlier studies. For instance, Borghans et al. (1996) showed that the MM kinetics is a good approximation to the quadratic kinetics when enzyme concentration is far smaller than the sum of the substrate concentration and the Michaelis-Menten constant (Palsson, 1987; Segel, 1988; Segel and Slemrod, 1989). To handle enzyme-substrate interactions under high enzyme concentrations, Borghans et al. (1996) proposed the total quasi-steady-state approximation (tQSSA) and obtained a substrate kinetics that was a special case of the later proposed equilibrium chemistry approximation (ECA) kinetics by Tang and Riley (2013). Tang and Riley (2013) applied the law of mass action with tQSSA and derived the ECA kinetics to describe the formation of enzyme-substrate complexes in a network of an arbitrary number of enzymes and substrates.

The consistent application of mathematical formulations to describe a dynamic system is critical for the model to successfully resolve the empirical measurements that observe the dynamic system. This consistency requirement has been raised in several studies using microbe explicit models. For instance, Maggi and Riley (2009) have found the MM kinetics has to be revised to resolve the evolution of $\delta^{15} \mathrm{~N}-\mathrm{N}_{2} \mathrm{O}$ in their data of nitrification and denitrification. Druhan et al. (2012) later used Maggi and Riley (2009)'s revision to obtain an improved modeling of the $\delta^{34} \mathrm{~S}$ data collected in the acetate-enabled uranium bioremediation at the US Department of Energy's Rifle Integrated Field Research Challenge site. Tang and Riley (2013) showed that the MM kinetics failed to resolve the evolution of the lignocellulose index during a litter decomposition experiment. I was not able to find any example of using the RMM kinetics to model kinetic isotope fractionation. However, because the RMM kinetics is a linear function of the substrate concentration, its application for modeling kinetic isotope fractionation will be doomed inevitably. Therefore, a substrate kinetics that merges the advantages from both the MM kinetics and the RMM kinetics would be a better choice for developing robust microbial models.

The call for a substrate kinetics that can consistently work across a wide range of substrate and enzyme (or more broadly competitor) concentrations becomes more imperative when land biogeochemical models are required to resolve plant-microbe interactions. In plant-microbe interactions, both substrates and competitors evolve constantly and their concentration ratios could vary by orders of magnitude. For instance, when a soil is seriously nitrogen limited, the aqueous nitrogen concentration is much lower than the volumetric density of competitors and substrate uptake may follow more linearly with respect to the substrate concentration and be of an asymptotic function of competitors as described by the RMM kinetics. However, when a large dose of fertilizer is added, the soil quickly becomes nitrogen saturated, such that the uptake dynamics would follow more linearly with respect to the variation of competitors (or enzymes) as represented in the MM kinetics. To consistently model the soil nitrogen dynamics that fluctuates between status of nitrogen limitation and nitrogen saturation, one therefore has to constantly choose between the MM kinetics and RMM kinetics, making a consistent mathematical formula- 
tion theoretically impossible. Therefore, an approach that includes the advantages from both the MM kinetics and RMM kinetics will significantly advance our capability in modeling soil biogeochemical processes. Fortunately, such kinetics (a.k.a. the ECA kinetics) was already derived in Tang and Riley (2013), but my coauthor and I did not give a theoretical analysis for the relationships between MM kinetics, RMM kinetics, and the ECA kinetics, nor did we explain how the parametric sensitivity would vary depending on the choice of substrate kinetics and whether the ECA kinetics is superior across the whole range of feasible kinetic parameters. Because all model calibration methods either explicitly or implicitly rely on the parametric sensitivity to tune model predictions with respect to observations (e.g., Tang and Zhuang, 2009; Zhu and Zhuang, 2014), correct parametric sensitivity of the model formulation is a requisite for delivering a robust model. An analysis of the differences in their predicted parametric sensitivities will also help to reveal the pitfalls that may exist in biogeochemical models that rely on the MM kinetics (Allison et al., 2010) or RMM kinetics (e.g., Averill, 2014) or a combination of the two (e.g., Sihi et al., 2015) when the model is otherwise benchmarked against its equilibrium-chemistry-based formulation that solves the biogeochemical system exactly under the tQSSA (readers please refer to Tang and Riley (2013) for a thorough discussion on why the equilibrium chemistry formulation should be the benchmark for models based on the MM kinetics, RMM kinetics, and ECA kinetics).

In this study, I first review how the ECA kinetics could be derived from the quadratic kinetics and how the MM kinetics and RMM kinetics could be derived from the ECA kinetics or directly from the equilibrium chemistry formulation of the enzyme-substrate interaction. Then I analyze how accurate the MM kinetics, the RMM kinetics, and the ECA kinetics could approximate the parametric sensitivity, as one would derive from the quadratic kinetics that is exact for the oneenzyme and one-substrate biogeochemical reaction. Based on these analyses, I finally give recommendations on how to obtain more robust microbial models for soil biogeochemical modeling. Note that, although the following analysis is for a single-enzyme and single-substrate system in an aqueous solution, the results are applicable to a wide range of problems, including predator-prey, microbial growth, Langmuir adsorption, and any process that can be appropriately formulated as an equilibrium binding problem (Tang and Riley, 2013).

\section{The mathematical relationship between different kinetics}

Below I first review how one could obtain the quadratic kinetics under the QSSA for a biogeochemical reaction that involves one enzyme and one substrate. Then I show how one could derive the ECA kinetics, the MM kinetics, and the RMM kinetics.

The biogeochemical reaction of the system is

$E+S \underset{k_{1}^{-}}{\stackrel{k_{1}^{+}}{\Leftrightarrow}} E S \stackrel{k_{2}^{+}}{\rightarrow} E+P$,

where $E, S, E S$, and $P$ are, respectively, enzyme, substrate, enzyme-substrate complex, and product. The three kinetic parameters are the intrinsic substrate affinity $k_{1}^{+}$ $\left(\mathrm{m}^{3} \mathrm{~mol}^{-1} \mathrm{~s}^{-1}\right)$, backward enzyme-substrate dissociation constant $k_{1}^{-}\left(\mathrm{s}^{-1}\right)$, and product genesis rate $k_{2}^{+}\left(\mathrm{s}^{-1}\right)$.

By the law of mass action, the governing equations for biogeochemical Reaction (1) are

$$
\begin{aligned}
& \frac{\mathrm{d}[E]}{\mathrm{d} t}=-k_{1}^{+}[S][E]+\left(k_{1}^{-}+k_{2}^{+}\right)[E S], \\
& \frac{\mathrm{d}[S]}{\mathrm{d} t}=-k_{1}^{+}[S][E]+k_{1}^{-}[E S], \\
& \frac{\mathrm{d}[E S]}{\mathrm{d} t}=k_{1}^{+}[S][E]-\left(k_{1}^{-}+k_{2}^{+}\right)[E S], \\
& \frac{\mathrm{d}[P]}{\mathrm{d} t}=k_{2}^{+}[E S] .
\end{aligned}
$$

Here and below, I use square brackets to designate the concentration $\left(\mathrm{mol} \mathrm{m}^{-3}\right)$ of a given state variable.

Under the QSSA, Eq. (4) is approximated as

$[S][E]=K_{E S}[E S]$,

where $K_{E S}=\left(k_{1}^{-}+k_{2}^{+}\right) / k_{1}^{+}\left(\mathrm{mol} \mathrm{m}^{-3}\right)$ is the MichaelisMenten constant.

For a small temporal window when the amount of new product is negligible, it holds that $[P] \ll[E S]+[S]=[S]_{T}$ (here and below subscript $T$ designates total concentration including both enzyme-substrate complex and free concentrations), then $[E S]$ could be solved from Eq. (6) under the constraints

$$
\begin{aligned}
& {[E S]+[E]=[E]_{T},} \\
& {[E S]+[S]=[S]_{T} .}
\end{aligned}
$$

By solving $[E]$ from Eq. (7), $[S]$ from Eq. (8) and entering the results into Eq. (6), one then obtains the quadratic equation

$$
[E S]^{2}-\left(K_{E S}+[E]_{T}+[S]_{T}\right)[E S]+[E]_{T}[S]_{T}=0 .
$$

Therefore, if one applies the quadratic formula to Eq. (9) and takes the physically meaningful solution, $[E S]$ is then found as

$$
\begin{aligned}
{[E S]=} & \frac{\left(K_{E S}+[E]_{T}+[S]_{T}\right)}{2} \\
& \left(1-\sqrt{1-\frac{4[E]_{T}[S]_{T}}{\left(K_{E S}+[E]_{T}+[S]_{T}\right)^{2}}}\right) .
\end{aligned}
$$




\subsection{The equilibrium chemistry approximation kinetics}

To obtain the ECA formulation of the enzyme-substrate complex, one assumes

$\varepsilon=\frac{[E]_{T}[S]_{T}}{\left(K_{E S}+[E]_{T}+[S]_{T}\right)^{2}} \ll 1$.

Then by substitution of the first-order approximation $\sqrt{1-4 \varepsilon} \approx(1-2 \varepsilon)$ (with Taylor expansion taken at $\varepsilon=0$; e.g., Cha and Cha, 1965) into the square root term of Eq. (10), the ECA formulation of $[E S]$ is obtained:

$[E S]=\frac{[E]_{T}[S]_{T}}{K_{E S}+[E]_{T}+[S]_{T}}$.

The application of Eq. (12) implies

$\frac{d[S]_{T}}{\mathrm{~d} t}=-k_{2}^{+}[E S]$,

which together with the QSSA forms the tQSSA (Borghans et al., 1996). Note Eq. (13) is more than a restatement of Eq. (5). Rather, Eq. (13) describes the temporal trend of the total substrate concentration instead of the temporal trend of the free substrate concentration, as done in the QSSA based MM kinetics shown below.

\subsection{The Michaelis-Menten kinetics}

The MM kinetics can be derived in two different approaches. In the first approach, by assuming $K_{E S}+[S]_{T} \gg[E]_{T}$, Eq. (12) gives the $\mathrm{MM}$ formulation of $[E S]$ :

$[E S] \approx \frac{[E]_{T}[S]_{T}}{K_{E S}+[S]_{T}}$.

In the second approach, one solves [ES] from Eqs. (6) and (7) and obtains

$[E S]=\frac{[E]_{T}[S]}{K_{E S}+[S]}$.

Note $[S]=[S]_{T}-[E S]<[S]_{T}$, and because $[E S]$ is a monotonically increasing function of $[S],[E S]$ computed from Eq. (14) will be greater than that from Eq. (15). However, almost all existing applications do not differentiate between Eqs. (14) and (15). The strict application of Eq. (14) requires the substrate evolution to be computed by the tQSSA form Eq. (13), whereas under the QSSA the strict application of Eq. (15) requires

$\frac{d[S]}{d t}=-k_{2}^{+}[E S]$.

When $[S]$ is low or when enzyme concentration $[E]_{T}$ is high, equating $[S]$ to $[S]_{T}$ and ignoring the contribution of $[E]_{T}$ in calculating the enzyme-substrate complex $[E S]$ will cause significant error in computing the parametric sensitivities, as I will show in Sect. 3.
The sufficient condition $K_{E S}+[S]_{T} \gg[E]_{T}$ (which always leads to $\varepsilon \ll 1$, the sufficient condition to derive the ECA kinetics) for the MM kinetics to be applicable was well recognized in early studies; however, it was often misinterpreted as $[S]_{T} \gg[E]_{T}$ (see a discussion in Borghans et al., 1996). Yet, more importantly, I note that the derivation of the MM kinetics does not take into account the mass balance constraint for substrate (Eq. 8). As I will show in Sect. 3, the negligence of mass balance constraint for substrate will lead to poor predictions of parametric sensitivity by the MM kinetics when benchmarked with the quadratic kinetics.

\subsection{The reverse Michaelis-Menten kinetics}

There are also two approaches to derive the RMM kinetics. In the first approach, one assumes $K_{E S}+[E]_{T} \gg[S]_{T}$ and then, from Eq. (12), obtains the RMM formulation of $[E S]$ :

$[E S] \approx \frac{[E]_{T}[S]_{T}}{K_{E S}+[E]_{T}}$.

In the second approach, one solves [ES] from Eqs. (6) and (8):

$[E S]=\frac{[E][S]_{T}}{K_{E S}+[E]}$.

Note $[E]=[E]_{T}-[E S]<[E]_{T}$ and, because $[E S]$ is a monotonically increasing function of $[E],[E S]$ calculated from Eq. (17) will be greater than that from Eq. (18). Like the MM kinetics, existing applications have treated Eqs. (17) and (18) as equivalent.

Here the condition $K_{E S}+[E]_{T} \gg[S]_{T}$ (which always leads to $\varepsilon \ll 1$, the sufficient condition to derive the ECA kinetics) for the RMM kinetics to hold is more general than the condition $[E]_{T} \gg[S]_{T}$ proposed in Wang and Post (2013). I also note that the derivation of the RMM kinetics does not take into account the mass balance constraint for enzymes (Eq. 7). This negligence of the mass balance constraint for enzymes will lead the RMM kinetics to predict poor parametric sensitivities when benchmarked with the quadratic kinetics.

\section{Parametric sensitivity analyses}

In the following I analyze the sensitivities of the reaction velocity with respect to the four parameters as predicted by the four kinetics. The four parameters are (1) maximum product genesis rate $k_{2}^{+},(2)$ intrinsic substrate affinity $k_{1}^{+}$, (3) the total enzyme concentration $[E]_{T}$, and (4) the total substrate concentration $[S]_{T}$. The reaction velocities predicted by the four different kinetics are, respectively, for the quadratic kinetics

$$
v_{\mathrm{QD}}=\frac{k_{2}^{+}\left(K_{E S}+[E]_{T}+[S]_{T}\right)}{2},\left(1-\sqrt{1-\frac{4[E]_{T}[S]_{T}}{\left(K_{E S}+[E]_{T}+[S]_{T}\right)^{2}}}\right),
$$


for the ECA kinetics

$v_{\mathrm{ECA}}=\frac{k_{2}^{+}[E]_{T}[S]_{T}}{K_{E S}+[E]_{T}+[S]_{T}}$,

for the MM kinetics

$v_{\mathrm{MM}}=\frac{k_{2}^{+}[E]_{T}[S]_{T}}{K_{E S}+[S]_{T}}$,

and for the RMM kinetics

$v_{\mathrm{RMM}}=\frac{k_{2}^{+}[E]_{T}[S]_{T}}{K_{E S}+[E]_{T}}$.

In evaluating the parametric sensitivity, I made the conventional assumption that $k_{1}^{-} \ll k_{2}^{+}$to obtain a better presentation of the results (although excluding this assumption will not change the conclusion below). This assumption leads to $K_{E S}=k_{2}^{+} / k_{1}^{+}$, which states that the apparent substrate affinity $1 / K_{E S}$ is a linearly decreasing function of $k_{2}^{+}$, a relationship that has been used to characterize the $K-r$ tradeoff (e.g., Litchman and Klausmeier, 2008). Because $K_{E S}$ is a function of $k_{2}^{+}$, the intrinsic affinity $k_{1}^{+}$better describes the substrate affinity for the enzymes.

In addition, to simplify the presentation, I define $y=$ $K_{E S}+[E]_{T}+[S]_{T}$ and $x=4[E]_{T}[S]_{T} / y^{2}$. Since the derivations for the MM- and RMM-kinetics-related parametric sensitivities could be derived from the ECA predictions straightforwardly, I only provide details to derive the results for the quadratic and ECA-related parametric sensitivities (Appendices A and B). Nevertheless, to help the readers to visualize the differences in the predicted parametric sensitivities by using different kinetics, I have summarized the comparison in four different figures: Fig. 1 for $k_{2}^{+}$, Fig. 2 for $k_{1}^{+}$, Fig. 3 for $[E]_{T}$, and Fig. 4 for $[S]_{T}$. All sensitivities are evaluated over the 2-D normalized substrate-enzyme concentration domain $[0.001,1000] \times[0.001,1000]$, with both $[E]_{T}$ and $[S]_{T}$ normalized by $K_{E S}$. In addition, because the quadratic kinetics is exact under the QSSA, its predictions are used to benchmark the predictions made by the ECA kinetics, MM kinetics, and RMM kinetics (see (d) panels in Figs. 1-4). For comparison between predictions by the ECA kinetics and the quadratic kinetics, I plotted the normalized sensitivities as 2-D functions of the normalized substrate $[S]_{T} / K_{E S}$ and $[E]_{T} / K_{E S}$ (see (a) and (b) panels in Figs. 1-4), and evaluated their differences using the index $\left(a_{\mathrm{QD}}-a_{\mathrm{ECA}}\right) /\left(a_{\mathrm{QD}}+a_{\mathrm{ECA}}\right)$ (see (c) panels in Figs. 1-4), where the subscripts QD and ECA indicate, respectively, sensitivities predicted by the quadratic kinetics and the ECA kinetics.

In all the analyses below, I represent the parametric sensitivity using the normalized form $\partial \ln v / \partial \ln s$ to remove the unit dependency of the results. The normalized sensitivity represents the relative change of reaction velocity $v$ in response to a relative change in parameter $s$, where $s$ could be any of the four parameters being analyzed.

\subsection{Reaction velocity vs. $k_{2}^{+}$}

The normalized sensitivities of the reaction velocity vs. $k_{2}^{+}$ are, respectively, for the quadratic kinetics

$$
\begin{aligned}
\frac{k_{2}^{+}}{v_{\mathrm{QD}}} \frac{\partial v_{\mathrm{QD}}}{\partial k_{2}^{+}}= & 1+\frac{K_{E S}}{y}-\frac{K_{E S}}{y}(1-\sqrt{1-x})^{-1} \\
& (1-x)^{-1 / 2} x,
\end{aligned}
$$

for the ECA kinetics

$\frac{k_{2}^{+}}{v_{\mathrm{ECA}}} \frac{\partial v_{\mathrm{ECA}}}{\partial k_{2}^{+}}=1-\frac{K_{E S}}{K_{E S}+[E]_{T}+[S]_{T}}$,

for the MM kinetics

$\frac{k_{2}^{+}}{v_{\mathrm{MM}}} \frac{\partial v_{\mathrm{MM}}}{\partial k_{2}^{+}}=1-\frac{K_{E S}}{K_{E S}+[S]_{T}}$,

and for the RMM kinetics

$\frac{k_{2}^{+}}{v_{\mathrm{RMM}}} \frac{\partial v_{\mathrm{RMM}}}{\partial k_{2}^{+}}=1-\frac{K_{E S}}{K_{E S}+[E]_{T}}$.

From the equations above, it is observed that both the MM kinetics and RMM kinetics predict a less variable and lower parametric sensitivity than the ECA kinetics, because the ECA kinetics predicts a more variable and larger denominator in the second term (in Eq. 24) as compared to that by the MM kinetics (Eq. 25) and RMM kinetics (Eq. 26). Large deviations between predicted sensitivities by the MM kinetics and the ECA kinetics are expected at high enzyme concentrations, whereas large deviations between predictions by the RMM kinetics and ECA kinetics are expected at high substrate concentrations. Predicted sensitivities by the MM kinetics and RMM kinetics are also smaller than those by the quadratic kinetics (green and black dots in Fig. 1d). In contrast, the ECA kinetics consistently captures the variability of the normalized sensitivity, with some overestimation (but the relative difference is no greater than $5 \%$ ) under moderate enzyme and substrate concentrations (Fig. 1c), where the normalized sensitivity is, however, small or moderate (Fig. 1a).

\subsection{Reaction velocity vs. $k_{1}^{+}$}

The normalized sensitivities of the reaction velocity vs. $k_{1}^{+}$ are, respectively, for the quadratic kinetics

$\frac{k_{1}^{+}}{v_{\mathrm{QD}}} \frac{\partial v_{\mathrm{QD}}}{\partial k_{1}^{+}}=-\frac{K_{E S}}{y}+\frac{K_{E S}}{y}(1-x)^{-1 / 2}(1-\sqrt{1-x})^{-1} x$,

for the ECA kinetics

$\frac{k_{1}^{+}}{v_{\mathrm{ECA}}} \frac{\partial v_{\mathrm{ECA}}}{\partial k_{1}^{+}}=\frac{K_{E S}}{K_{E S}+[E]_{T}+[S]_{T}}$,

for the MM kinetics

$\frac{k_{1}^{+}}{v_{\mathrm{MM}}} \frac{\partial v_{\mathrm{MM}}}{\partial k_{1}^{+}}=\frac{K_{E S}}{K_{E S}+[S]_{T}}$, 

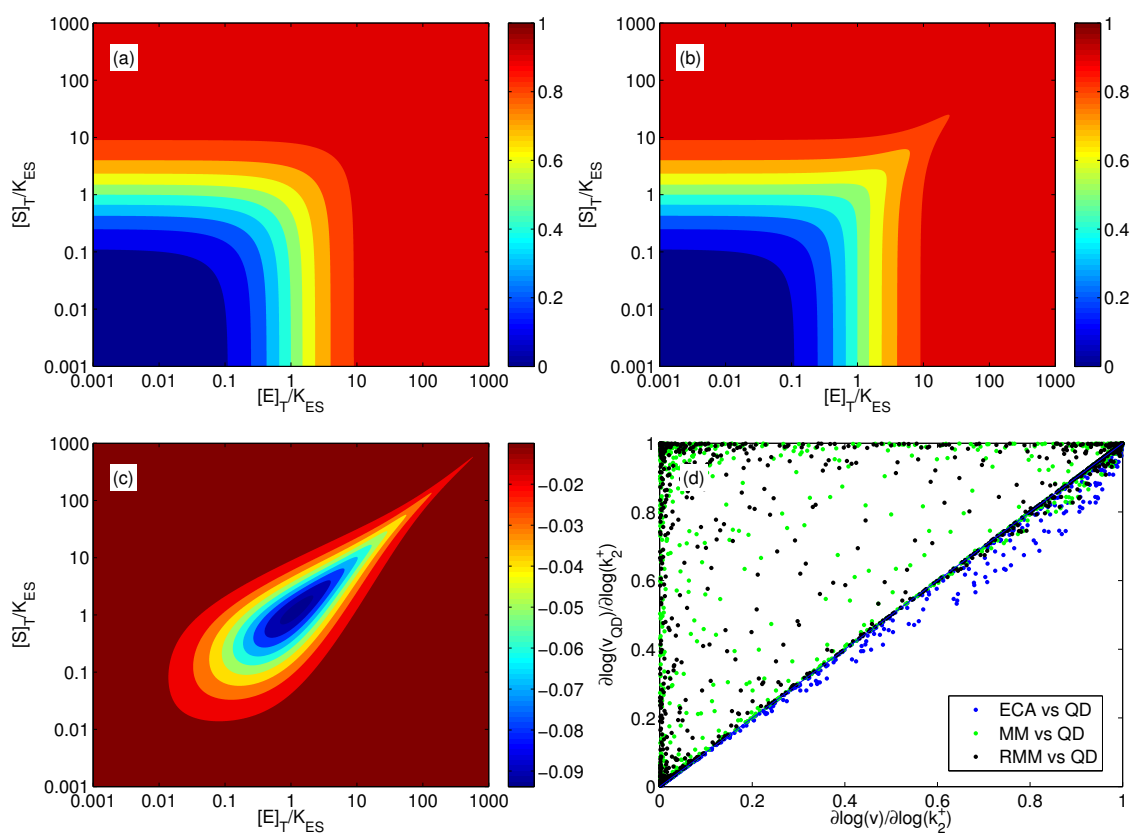

Figure 1. (a) ECA-kinetics-predicted normalized sensitivity of the reaction velocity with respect to the maximum product genesis rate $k_{2}^{+}$; (b) predictions by the quadratic kinetics; (c) the normalized difference $\left(a_{\mathrm{QD}}-a_{\mathrm{ECA}}\right) /\left(a_{\mathrm{QD}}+a_{\mathrm{ECA}}\right)$ between the quadratic kinetics predictions $a_{\mathrm{QD}}$ and the ECA kinetics predictions $a_{\mathrm{ECA}} ;(\mathbf{d})$ comparison of normalized sensitivity predicted by different kinetics.

and for the RMM kinetics

$$
\frac{k_{1}^{+}}{v_{\mathrm{RMM}}} \frac{\partial v_{\mathrm{RMM}}}{\partial k_{1}^{+}}=\frac{K_{E S}}{K_{E S}+[E]_{T}} \text {. }
$$

From Eqs. (28)-(30), it is inferred that both the MM kinetics and RMM kinetics predict a less variable and higher normalized sensitivity with respect to $k_{1}^{+}$than the ECA kinetics. Large differences between predicted sensitivities by the ECA kinetics and the MM kinetics are expected at high enzyme concentrations, whereas large differences between predicted sensitivities by the ECA kinetics and the RMM kinetics are expected at high substrate concentrations. The predicted sensitivities by the MM kinetics and RMM kinetics are also lower than that by the quadratic kinetics (Fig. 2d), whereas the ECA kinetics predicts consistent parametric sensitivity for the wide range of enzyme and substrate concentrations (Fig. 2). The underpredicted sensitivity by the ECA kinetics is significant only at high substrate and high enzyme concentrations (Fig. 2c), where the parametric sensitivity is close to zero (Fig. 2a, b).

\subsection{Reaction velocity vs. $[E]_{T}$}

The normalized sensitivities of the reaction velocity vs. $[E]_{T}$ are, respectively, for the quadratic kinetics

$$
\begin{aligned}
& \frac{[E]_{T}}{v_{\mathrm{QD}}} \frac{\partial v_{\mathrm{QD}}}{\partial[E]_{T}}=\frac{[E]_{T}}{y}+\frac{[E]_{T}}{y} \\
& (1-\sqrt{1-x})^{-1}(1-x)^{-1 / 2} \times\left(\frac{2[S]_{T}}{y}-x\right),
\end{aligned}
$$

for the ECA kinetics

$$
\frac{[E]_{T}}{v_{\mathrm{ECA}}} \frac{\partial v_{\mathrm{ECA}}}{\partial[E]_{T}}=1-\frac{[E]_{T}}{K_{E S}+[E]_{T}+[S]_{T}},
$$

for the MM kinetics

$\frac{[E]_{T}}{v_{\mathrm{MM}}} \frac{\partial v_{\mathrm{MM}}}{\partial[E]_{T}}=1$,

and for the RMM kinetics

$\frac{[E]_{T}}{v_{\mathrm{RMM}}} \frac{\partial v_{\mathrm{RMM}}}{\partial[E]_{T}}=1-\frac{[E]_{T}}{K_{E S}+[E]_{T}}$.

From the equations above, it is observed that the MM kinetics predicts a constant normalized sensitivity of the reaction velocity with respect to the total enzyme concentration $[E]_{T}$. The RMM kinetics predicts the normalized sensitivity as a monotonically decreasing function of the normalized enzyme concentration $[E]_{T} / K_{E S}$. The predicted sensitivity by the ECA kinetics is a function of both the normalized substrate concentration $[S]_{T} / K_{E S}$ and the normalized enzyme concentration $[E]_{T} / K_{E S}$. Compared to predictions by the quadratic kinetics, the MM kinetics persistently overestimates the parametric sensitivity (green dots in Fig. 3d), whereas the RMM kinetics persistently underestimates the parametric sensitivity (black dots in Fig. 3d). The ECA predicted sensitivity is largely consistent with that by the quadratic kinetics (Fig. 3), albeit with some significant deviation in regions of very high substrate and enzyme concentrations (Fig. 3c), where the parametric uncertainty is moderate or low (Fig. 3a, b). 

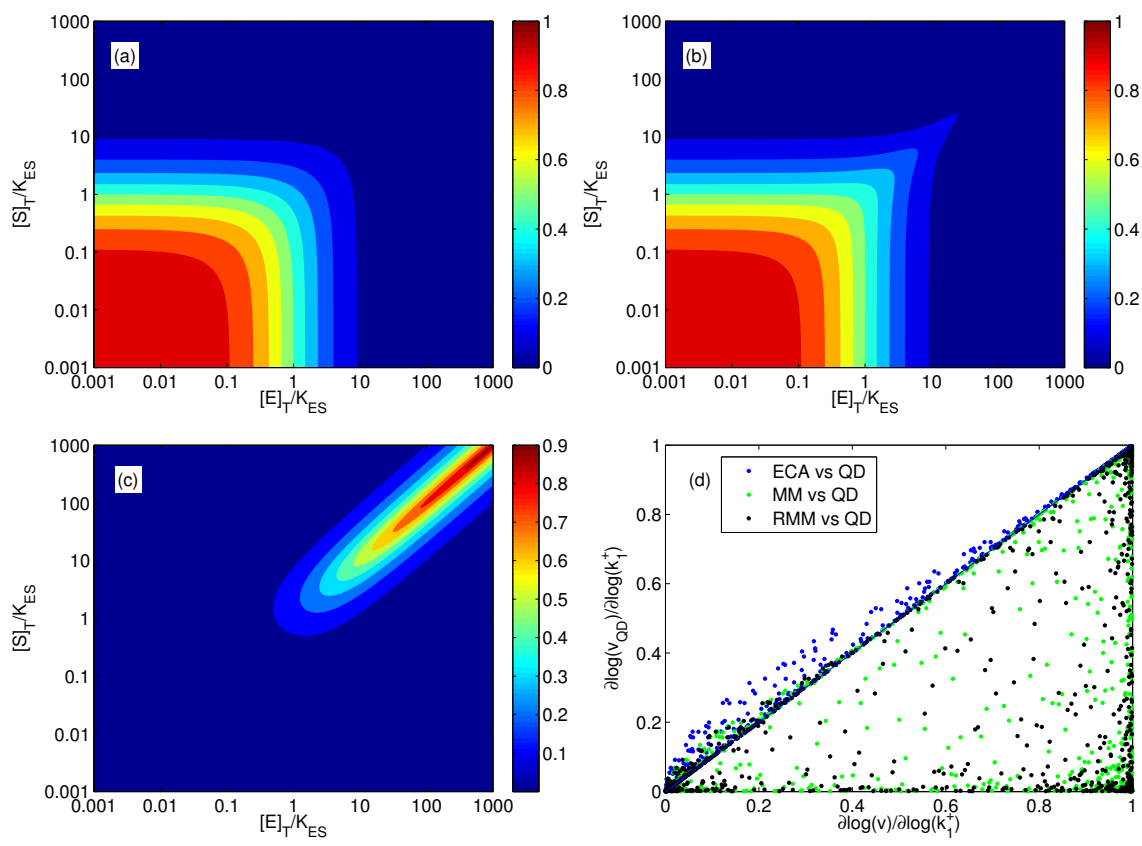

Figure 2. Similar to Fig. 1, but the sensitivity is evaluated against the intrinsic substrate affinity $k_{1}^{+}$.

\subsection{Reaction velocity vs. $[S]_{T}$}

The normalized sensitivities of the reaction velocity vs. $[S]_{T}$ are, respectively, for the quadratic kinetics

$$
\begin{aligned}
& \frac{[S]_{T}}{v_{\mathrm{QD}}} \frac{\partial v_{\mathrm{QD}}}{\partial[S]_{T}}=\frac{[S]_{T}}{y}+\frac{[S]_{T}}{y}(1-\sqrt{1-x})^{-1} \\
& (1-x)^{-1 / 2} \times\left(\frac{2[E]_{T}}{y}-x\right),
\end{aligned}
$$

for the ECA kinetics

$$
\frac{[S]_{T}}{v_{\mathrm{ECA}}} \frac{\partial v_{\mathrm{ECA}}}{\partial[S]_{T}}=1-\frac{[S]_{T}}{K_{E S}+[E]_{T}+[S]_{T}},
$$

for the MM kinetics

$$
\frac{[S]_{T}}{v_{\mathrm{MM}}} \frac{\partial v_{\mathrm{MM}}}{\partial[S]_{T}}=1-\frac{[S]_{T}}{K_{E S}+[S]_{T}},
$$

and for the RMM kinetics

$$
\frac{[S]_{T}}{v_{\mathrm{RMM}}} \frac{\partial v_{\mathrm{RMM}}}{\partial[S]_{T}}=1 \text {. }
$$

Because $[S]_{T}$ and $[E]_{T}$ are symmetric in the quadratic kinetics and ECA kinetics, the predicted normalized sensitivity of the reaction velocity with respect to the total substrate concentration $[S]_{T}$ mirrors that of $[E]_{T}$ along the lower-left to upper-right diagonal (Fig. 3 vs. Fig. 4). Such symmetric relationships also exist in predictions by the MM kinetics and RMM kinetics; however, the MM kinetics persistently underpredicts the normalized sensitivity of the reaction velocity with respect to $[S]_{T}$, and the RMM kinetics predicts a constant sensitivity (Eq. 38). The ECA kinetics once again predicts consistent parametric sensitivity when compared with the quadratic kinetics.

\section{Discussions and conclusions}

From the above analyses, I showed that the ECA kinetics is a better approximation to the quadratic kinetics, which, obtained from the law of mass action and the quasi-steadystate approximation, is the exact solution to the governing equations of substrate-enzyme interaction (as indicated by Eqs. 6-8). In contrast, the Michaelis-Menten kinetics and the reverse Michaelis-Menten kinetics are inferior in approximating the quadratic kinetics over the wide range of enzyme and substrate concentrations. The worse performance of the MM kinetics than the ECA kinetics in approximating the quadratic kinetics stems from the negligence of mass balance constraint of the substrate during the derivation of the MM kinetics; while the worse performance of the RMM kinetics in approximating the quadratic kinetics is caused by the negligence of mass balance constraint of the enzyme during the derivation of the RMM kinetics. The failure to consider the mass balance constraints for both enzyme and substrate during their derivations caused the MM kinetics and the RMM kinetics to predict significantly biased normalized sensitivity of the reaction velocity with respect to the two kinetic parameters $k_{1}^{+}$and $k_{2}^{+}$, the total enzyme concentration $[E]_{T}$, and the total substrate concentration $[S]_{T}$. Although being a first-order approximation to the quadratic kinetics under 

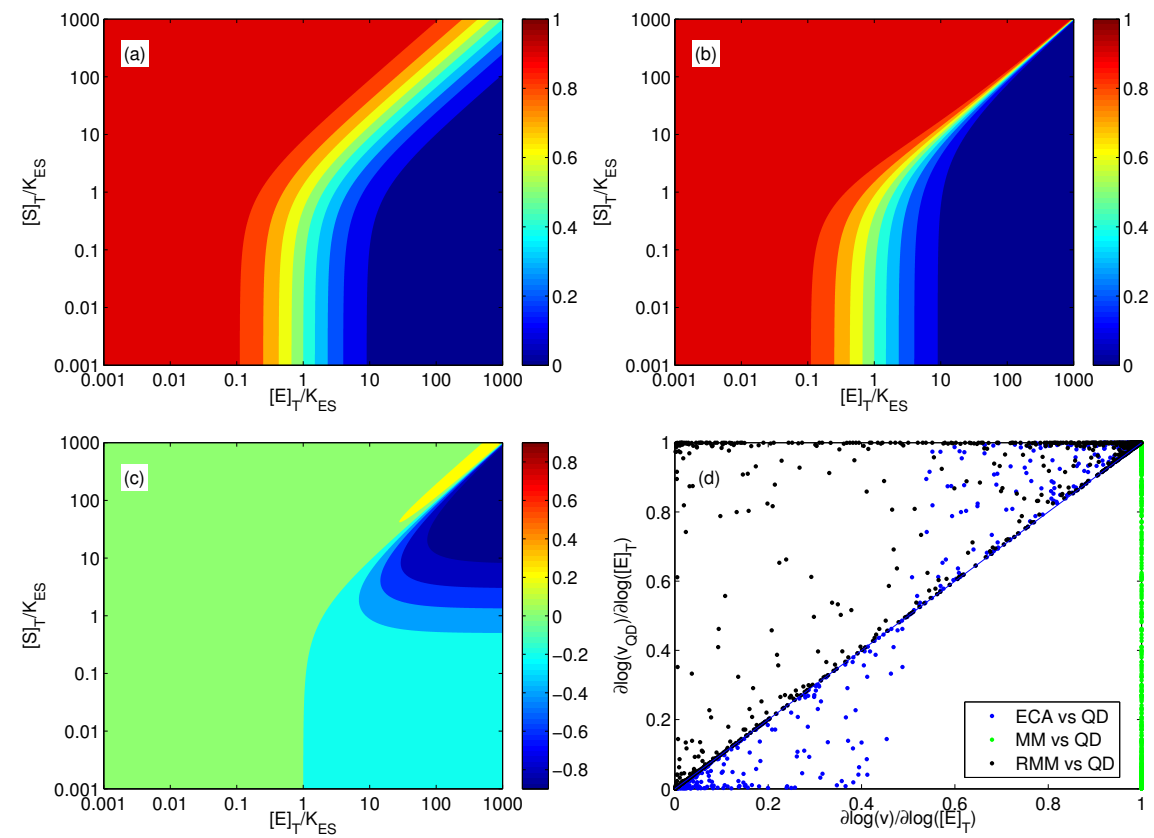

Figure 3. Similar to Fig. 1, but the sensitivity is evaluated against the total enzyme concentration $[E]_{T}$.
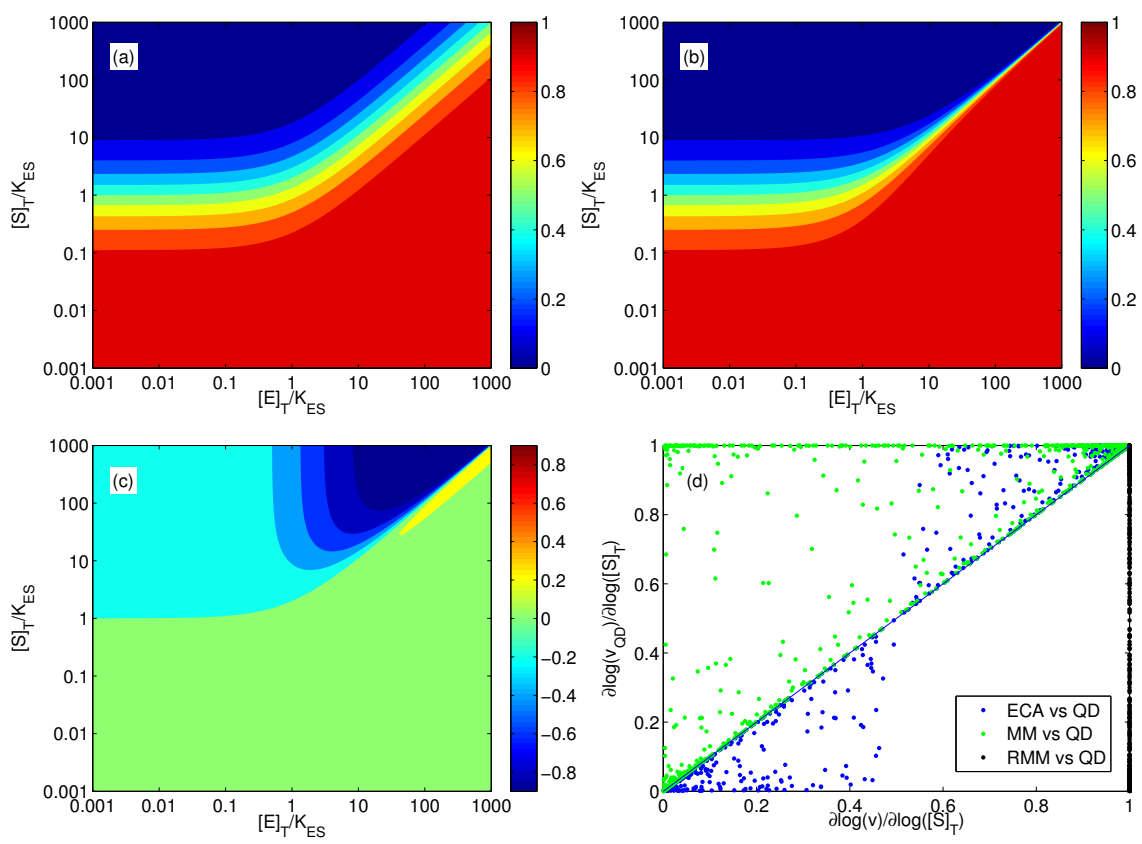

Figure 4. Similar to Fig. 1, but the sensitivity is evaluated against the total substrate concentration $[S]_{T}$.

the assumption that $[E]_{T}[S]_{T} \ll\left(K_{E S}+[E]_{T}+[S]_{T}\right)^{2}$, because it considers the mass balance for both substrate and enzyme, the ECA kinetics predicts consistent parametric sensitivity with that by the quadratic kinetics over the wide range of normalized substrate and enzyme concentrations.

In modeling complex soil biogeochemical dynamics, the consistency between the kinetics used and the equilibrium chemistry formulation of the relationships between enzymes, substrates, and enzyme-substrate complexes might be critical (Tang and Riley, 2013), but it has been unfortunately underappreciated in many previous studies. In Tang and Riley (2013), it was shown that, for a system involving three microbes competitively decomposing three carbon substrates, the MM kinetics failed wildly even with industrious calibration (see their Fig. 12). In an earlier study, Moorhead and Sinsabaugh (2006) had to prescribe the relative decom- 
position between lignin and cellulose in order to resolve the lignocellulose index dynamics. The ECA kinetics was able to consistently resolve the lignin-cellulose dynamics during the litter decomposition because it explicitly considers the mass balance constraints for each of the substrates and enzymes (or, effectively, abundance of competitors; Tang and Riley, 2013). Both the success of the ECA kinetics and the failure of the MM kinetics in abovementioned studies can be traced back to their capability in approximating the actual parametric sensitivities of the specific dynamic system. Because all model calibration techniques rely on a model's parametric sensitivity to obtain improved agreement between model predictions and measurements, wrong parametric sensitivity as formulated in the adopted substrate kinetics would result in a non-calibratable or poorly calibratable model, which could be manifested as systematic model biases or completely unreasonable model predictions. This explained well why the MM-kinetics-based model in Tang and Riley (2013) failed wildly even with intensive Bayesian model calibration.

Therefore, if the ecological dynamics involved in substrate processing by microbes does approximately obey the law of mass action and the total quasi-steady-state approximation (as it is already implied in any microbe explicit model that uses the MM kinetics or the RMM kinetics), then the analytically tractable ECA kinetics is a much more powerful and mathematically more consistent tool than the popular MM kinetics and RMM kinetics that are currently used in many microbial models. Indeed, a recent application (Zhu and Riley, 2015) indicated that by representing plant-microbe competition of soil mineral nitrogen using the ECA kinetics, the predicted global nitrogendynamics became much more consistent with that inferred from the $\delta^{15} \mathrm{~N}$ isotopic data (Houlton et al., 2015). The ECA kinetics was also found to satisfy- ingly model the plant-microbe competitions for phosphorus and mineral nitrogen at several fertilized sites (Zhu et al., 2015a) and predicted consistent vertical nitrogen uptake profiles measured at an alpine meadow ecosystem (Zhu et al., 2015b). Theoretically, because either the MM kinetics or the RMM kinetics works only in a small subdomain of the parameters that are used in the original quadratic kinetics, models based on the MM kinetics or RMM kinetics may likely have a much lower predictive capability than is implied in the mechanisms that the models are trying to represent (e.g., the law of mass action, which is the foundation to all substrate kinetics). I therefore recommend modelers to use the ECA kinetics to describe the substrate uptake processes in modeling microbe-regulated biogeochemical processes. As I showed above, with the same number of parameters as one would use with either the MM kinetics or the RMM kinetics, the ECA kinetics achieved better accuracy in approximating the exact quadratic kinetics for a biogeochemical reaction that involves a single enzyme and a single substrate. The superior performance of ECA is also true for systems that involve many substrates and many enzymes (Tang and Riley, 2013), which are much more common in the natural environment that we are trying to model. Lastly, and more importantly, the ECA kinetics could spare modelers from the pain of deciding when to use the MM kinetics or the RMM kinetics to represent a soil that fluctuates between status of nutrient limitation and nutrient saturation, for which neither the MM kinetics nor the RMM kinetics is (but ECA is) theoretically consistent with the law of mass action, the best theory we have for modeling biogeochemical reactions. 
Appendix A: Derivation of parametric sensitivities (Eqs. 23, 27, 31, and 35) for the quadratic kinetics

Using the definitions $y=K_{E S}+[E]_{T}+[S]_{T}$ and $x=$ $4[E]_{T}[S]_{T} / y^{2}$, one has the following results:

$$
\begin{aligned}
& v_{\mathrm{QD}}=\frac{k_{2}^{+} y}{2}(1-\sqrt{1-x}), \\
& \frac{\partial x}{\partial k_{1}^{+}}=\frac{8[E]_{T}[S]_{T}}{\left(K_{E S}+[E]_{T}+[S]_{T}\right)^{3}} \frac{K_{E S}}{k_{1}^{+}}=\frac{8[E]_{T}[S]_{T}}{y^{3}} \frac{K_{E S}}{k_{1}^{+}}, \\
& \frac{\partial x}{\partial k_{2}^{+}}=-\frac{8[E]_{T}[S]_{T}}{\left(K_{E S}+[E]_{T}+[S]_{T}\right)^{3}} \frac{1}{k_{1}^{+}}=-\frac{8[E]_{T}[S]_{T}}{y^{3}} \frac{1}{k_{1}^{+}}, \\
& \frac{\partial x}{\partial[E]_{T}}=\frac{4[S]_{T}}{\left(K_{E S}+[S]_{T}+[E]_{T}\right)^{2}}- \\
& \frac{8[E]_{T}[S]_{T}}{\left(K_{E S}+[S]_{T}+[E]_{T}\right)^{3}}=\frac{4[S]_{T}}{y^{2}}-\frac{2 x}{y}, \\
& \frac{\partial x}{\partial[S]_{T}}=\frac{4[E]_{T}}{\left(K_{E S}+[S]_{T}+[E]_{T}\right)^{2}}- \\
& \frac{8[E]_{T}[S]_{T}}{\left(K_{E S}+[S]_{T}+[E]_{T}\right)^{3}}=\frac{4[E]_{T}}{y^{2}}-\frac{2 x}{y}, \\
& \frac{\partial \sqrt{1-x}}{\partial k_{1}^{+}}=-\frac{1}{2}(1-x)^{-1 / 2} \frac{\partial x}{\partial k_{1}^{+}}, \\
& \frac{\partial \sqrt{1-x}}{\partial k_{2}^{+}}=-\frac{1}{2}(1-x)^{-1 / 2} \frac{\partial x}{\partial k_{2}^{+}}, \\
& \frac{\partial \sqrt{1-x}}{\partial[E]_{T}}=-\frac{1}{2}(1-x)^{-1 / 2} \frac{\partial x}{\partial[E]_{T}}, \\
& \frac{\partial \sqrt{1-x}}{\partial[S]_{T}}=-\frac{1}{2}(1-x)^{-1 / 2} \frac{\partial x}{\partial[S]_{T}}, \\
& \frac{\partial y}{\partial k_{1}^{+}}=\frac{\partial K_{E S}}{\partial k_{1}^{+}}=-\frac{K_{E S}}{k_{1}^{+}}, \\
& \frac{\partial y}{\partial k_{2}^{+}}=\frac{\partial K_{E S}}{\partial k_{2}^{+}}=\frac{1}{k_{1}^{+}}, \\
& \frac{\partial y}{\partial[E]_{T}}=\frac{\partial y}{2[S]_{T}}=1 . \\
&
\end{aligned}
$$

Then, from Eq. (A1), one has

$$
\begin{aligned}
& \frac{\partial v_{\mathrm{QD}}}{\partial k_{2}^{+}}=\frac{y}{2}(1-\sqrt{1-x})+\frac{k_{2}^{+}}{2}(1-\sqrt{1-x}) \frac{\partial y}{\partial k_{2}^{+}} \\
& -\frac{k_{2}^{+}}{2} y \frac{\partial \sqrt{1-x}}{\partial k_{2}^{+}} .
\end{aligned}
$$

By substitution of Eqs. (A3), (A7), and (A11) into (A13), and using the definition of $v_{\mathrm{QD}}$ from Eq. (A1), one obtains

$$
\begin{aligned}
& \frac{\partial v_{\mathrm{QD}}}{\partial k_{2}^{+}}=\frac{y}{2}(1-\sqrt{1-x})+\frac{K_{E S}}{2}(1-\sqrt{1-x})-\frac{K_{E S}}{2}(1-x)^{-1 / 2} x \\
& =\frac{v_{\mathrm{QD}}}{k_{2}^{+}}\left\{1+\frac{K_{E S}}{y}-\frac{K_{E S}}{y}(1-\sqrt{1-x})^{-1}(1-x)^{-1 / 2} x\right\},
\end{aligned}
$$

which, after some rearrangements, gives Eq. (23) in the main text.

Similarly, from Eq. (A1), one has

$$
\frac{\partial v_{\mathrm{QD}}}{\partial k_{1}^{+}}=\frac{k_{2}^{+}}{2}(1-\sqrt{1-x}) \frac{\partial y}{\partial k_{1}^{+}}-\frac{k_{2}^{+} y}{2} \frac{\partial \sqrt{1-x}}{\partial k_{1}^{+}},
$$

which, after using Eqs. (A2), (A6), and (A10), leads to

$$
\begin{aligned}
& \frac{\partial v_{\mathrm{QD}}}{\partial k_{1}^{+}}=-\frac{1}{2} K_{E S}^{2}(1-\sqrt{1-x})+\frac{1}{2} K_{E S}^{2}(1-x)^{-1 / 2} x \\
& =\frac{v_{\mathrm{QD}}}{k_{1}^{+}}\left\{-\frac{K_{E S}}{y}+\frac{K_{E S}}{y}(1-x)^{-1 / 2}(1-\sqrt{1-x})^{-1} x\right\} .
\end{aligned}
$$

By multiplying $k_{1}^{+} / v_{\mathrm{QD}}$ on both sides of Eq. (A16), one easily obtains Eq. (27).

Taking the partial derivative with respect to $[E]_{T}$ in Eq. (A1), one obtains

$$
\frac{\partial v_{\mathrm{QD}}}{\partial[E]_{T}}=\frac{k_{2}^{+}}{2}(1-\sqrt{1-x}) \frac{\partial y}{\partial[E]_{T}}-\frac{k_{2}^{+} y}{2} \frac{\partial \sqrt{1-x}}{\partial[E]_{T}},
$$

which, when combined with Eqs. (A4), (A8), and (A12), becomes

$$
\begin{aligned}
& \frac{\partial v_{\mathrm{QD}}}{\partial[E]_{T}}=\frac{k_{2}^{+}}{2}(1-\sqrt{1-x})+\frac{k_{2}^{+}}{2}(1-x)^{-1 / 2}\left(\frac{2[S]_{T}}{y}-x\right) \\
& =\frac{v_{\mathrm{QD}}}{[E]_{T}}\left\{\frac{[E]_{T}}{y}+\frac{[E]_{T}}{y}(1-\sqrt{1-x})^{-1}(1-x)^{-1 / 2}\right. \\
& \left.\times\left(\frac{2[S]_{T}}{y}-x\right)\right\},
\end{aligned}
$$

from which, after some rearrangement, one finds Eq. (31).

Note, because switching the order of $[E]_{T}$ and $[S]_{T}$ in Eq. (A1) does not change the definition of $v_{\mathrm{QD}}$, Eq. (35) could be derived from Eq. (31) by simply swapping $[E]_{T}$ and $[S]_{T}$.

\section{Appendix B: Derivation of parametric sensitivities} (Eqs. 24, 28, 32, and 36) for the equilibrium chemistry approximation kinetics

Using the definitions of $x$ and $y, v_{\mathrm{ECA}}$ is

$v_{\mathrm{ECA}}=\frac{k_{2}^{+}[E]_{T}[S]_{T}}{y}$.

From Eq. (B1), one has

$\frac{\partial v_{\mathrm{ECA}}}{\partial k_{2}^{+}}=\frac{[E]_{T}[S]_{T}}{y}-\frac{k_{2}^{+}[E]_{T}[S]_{T}}{y^{2}} \frac{\partial y}{\partial k_{2}^{+}}$,

which, when combined with Eq. (A11), becomes

$\frac{\partial v_{\mathrm{ECA}}}{\partial k_{2}^{+}}=\frac{v_{\mathrm{ECA}}}{k_{2}^{+}}-\frac{v_{\mathrm{ECA}}}{k_{2}^{+}} \frac{K_{E S}}{y}$. 
Then, by dividing both sides of Eq. (B3) with $v_{\mathrm{ECA}} / k_{2}^{+}$, one obtains Eq. (24).

Similarly, from Eq. (B1), one has

$\frac{\partial v_{\mathrm{ECA}}}{\partial k_{1}^{+}}=-\frac{k_{2}^{+}[E]_{T}[S]_{T}}{y^{2}} \frac{\partial y}{\partial k_{1}^{+}}$.

Then, by aid of Eq. (A10), one finds

$\frac{\partial v_{\mathrm{ECA}}}{\partial k_{1}^{+}}=\frac{v_{\mathrm{ECA}}}{k_{1}^{+}} \frac{K_{E S}}{y}$,

which gives Eq. (28) by multiplying $k_{1}^{+} / v_{\mathrm{ECA}}$ on both sides.

For $[E]_{T}$, one can derive from Eq. (B1)

$\frac{\partial v_{\mathrm{ECA}}}{\partial[E]_{T}}=\frac{k_{2}^{+}[S]_{T}}{y}-\frac{k_{2}^{+}[E]_{T}[S]_{T}}{y^{2}} \frac{\partial y}{\partial[E]_{T}}$,

which, when combined with Eq. (A12), leads to

$\frac{\partial v_{\mathrm{ECA}}}{\partial[E]_{T}}=\frac{v_{\mathrm{ECA}}}{[E]_{T}}-\frac{v_{\mathrm{ECA}}}{y}$.

Then, by dividing both sides of Eq. (B7) with $v_{\mathrm{ECA}} /[E]_{T}$, one obtains Eq. (32).

By using the symmetry between $[E]_{T}$ and $[S]_{T}$ in the definition of $v_{\mathrm{ECA}}$, Eq. (36) could be obtained by swapping [E] $]_{T}$ and $[S]_{T}$ in Eq. (32). 
Author contributions. J. Y. Tang developed the theory, conducted the analyses, and wrote the paper.

Acknowledgements. This research is supported by the Director, Office of Science, Office of Biological and Environmental Research of the US Department of Energy under contract no. DE-AC02-05CH11231 as part of the Next-Generation Ecosystem Experiments (NGEE-Arctic) and the Accelerated Climate Model for Energy project in the Earth System Modeling program. I sincerely thank Thomas Wutzler, Joshua Schimel, an anonymous reviewer, and the handling editor for their constructive comments, which significantly improved the paper.

Edited by: C. Sierra

\section{References}

Allison, S. D., Wallenstein, M. D., and Bradford, M. A.: Soil-carbon response to warming dependent on microbial physiology, Nat. Geosci., 3, 336-340, 2010.

Averill, C.: Divergence in plant and microbial allocation strategies explains continental patterns in microbial allocation and biogeochemical fluxes, Ecol. Lett., 17, 1202-1210, 2014.

Borghans, J. A. M., DeBoer, R. J., and Segel, L. A.: Extending the quasi-steady state approximation by changing variables, B Math. Biol., 58, 43-63, 1996.

Cha, S. and Cha, C. J. M.: Kinetics of Cyclic Enzyme Systems, Mol. Pharmacol., 1, 178-189, 1965.

Drake, J. E., Darby, B. A., Giasson, M.-A., Kramer, M. A., Phillips, R. P., and Finzi, A. C.: Stoichiometry constrains microbial response to root exudation- insights from a model and a field experiment in a temperate forest, Biogeosciences, 10, 821-838, doi:10.5194/bg-10-821-2013, 2013.

Druhan, J. L., Steefel, C. I., Molins, S., Williams, K. H., Conrad, M. E., and DePaolo, D. J.: Timing the onset of sulfate reduction over multiple subsurface acetate amendments by measurement and modeling of sulfur isotope fractionation, Environ. Sci. Technol., 46, 8895-8902, doi:10.1021/Es302016p, 2012.

German, D. P., Marcelo, K. R. B., Stone, M. M., and Allison, S. D.: The Michaelis-Menten kinetics of soil extracellular enzymes in response to temperature: a cross-latitudinal study, Global Change Biol., 18, 1468-1479, 2012.

He, Y., Zhuang, Q., Harden, J. W., McGuire, A. D., Fan, Z., Liu, Y., and Wickland, K. P.: The implications of microbial and substrate limitation for the fates of carbon in different organic soil horizon types of boreal forest ecosystems: a mechanistically based model analysis, Biogeosciences, 11, 4477-4491, doi:10.5194/bg-114477-2014, 2014.

Houlton, B. Z., Marklein, A. R., and Bai, E.: Representation of nitrogen in climate change forecasts, Nat. Clim. Change, 5, 398401, 2015.

Johnson, F. H. and Lewin, I.: The Growth Rate of E-Coli in Relation to Temperature, Quinine and Coenzyme, J. Cell. Compar. Physl., 28, 47-75, 1946.

Li, J. W., Wang, G. S., Allison, S. D., Mayes, M. A., and Luo, Y. Q.: Soil carbon sensitivity to temperature and carbon use effi- ciency compared across microbial-ecosystem models of varying complexity, Biogeochemistry, 119, 67-84, 2014.

Litchman, E. and Klausmeier, C. A.: Trait-Based Community Ecology of Phytoplankton, Annu. Rev. Ecol. Evol. S, 39, 615-639, 2008.

Maggi, F. and Riley, W. J.: Transient competitive complexation in biological kinetic isotope fractionation explains nonsteady isotopic effects: Theory and application to denitrification in soils, J. Geophys. Res.-Biogeo., 114, G04012, doi:10.1029/2008jg000878, 2009.

Michaelis, L. and Menten, M. L.: The kenetics of the inversion effect, Biochem. Z., 49, 333-369, 1913.

Moorhead, D. L. and Sinsabaugh, R. L.: A theoretical model of litter decay and microbial interaction, Ecol. Monogr., 76, 151-174, 2006.

Palsson, B. O.: On the Dynamics of the Irreversible Michaelis Menten Reaction-Mechanism, Chem. Eng. Sci., 42, 447-458, 1987.

Riley, W. J., Maggi, F., Kleber, M., Torn, M. S., Tang, J. Y., Dwivedi, D., and Guerry, N.: Long residence times of rapidly decomposable soil organic matter: application of a multi-phase, multi-component, and vertically resolved model (BAMS1) to soil carbon dynamics, Geosci. Model Dev., 7, 1335-1355, doi:10.5194/gmd-7-1335-2014, 2014.

Schimel, J. P. and Weintraub, M. N.: The implications of exoenzyme activity on microbial carbon and nitrogen limitation in soil: a theoretical model, Soil Biol. Biochem., 35, 549-563, 2003.

Segel, L. A.: On the Validity of the Steady-State Assumption of Enzyme-Kinetics, B. Math. Biol., 50, 579-593, 1988.

Segel, L. A. and Slemrod, M.: The Quasi-Steady-State Assumption - a Case-Study in Perturbation, Siam Rev., 31, 446-477, 1989.

Sihi, D., Gerber, S., Inglett, P. W., and Inglett, K. S.: Comparing models of microbial-substrate interactions and their response to warming, Biogeosciences Discuss., 12, 10857-10897, doi:10.5194/bgd-12-10857-2015, 2015.

Sulman, B. N., Phillips, R. P., Oishi, A. C., Shevliakova, E., and Pacala, S. W.: Microbe-driven turnover offsets mineral-mediated storage of soil carbon under elevated $\mathrm{CO}_{2}$, Nat. Clim. Change, 4 , 1099-1102, 2014.

Tang, J. Y. and Zhuang, Q. L.: A global sensitivity analysis and Bayesian inference framework for improving the parameter estimation and prediction of a process-based Terrestrial Ecosystem Model, J. Geophys. Res.-Atmos., 114, D15303, doi:10.1029/2009JD011724, 2009.

Tang, J. Y. and Riley, W. J.: A total quasi-steady-state formulation of substrate uptake kinetics in complex networks and an example application to microbial litter decomposition, Biogeosciences, 10, 8329-8351, doi:10.5194/bg-10-8329-2013, 2013.

Tang, J. Y. and Riley, W. J.: Weaker soil carbon-climate feedbacks resulting from microbial and abiotic interactions, Nat. Clim. Change, 5, 56-60, 2015.

Wang, G. S. and Post, W. M.: A note on the reverse MichaelisMenten kinetics, Soil Biol. Biochem., 57, 946-949, 2013.

Wang, G. S., Post, W. M., and Mayes, M. A.: Development of microbial-enzyme-mediated decomposition model parameters through steady-state and dynamic analyses, Ecol. Appl., 23, 255$272,2013$.

Waring, B. G., Averill, C., and Hawkes, C. V.: Differences in fungal and bacterial physiology alter soil carbon and nitrogen cycling: 
insights from meta-analysis and theoretical models, Ecol. Lett., 16, 887-894, 2013.

Wieder, W. R., Bonan, G. B., and Allison, S. D.: Global soil carbon projections are improved by modelling microbial processes, Nat. Clim. Change, 3, 909-912, 2013.

Wieder, W. R., Grandy, A. S., Kallenbach, C. M., Taylor, P. G., and Bonan, G. B.: Representing life in the Earth system with soil microbial functional traits in the MIMICS model, Geosci. Model Dev., 8, 1789-1808, doi:10.5194/gmd-8-1789-2015, 2015.

Xenakis, G. and Williams, M.: Comparing microbial and chemical kinetics for modelling soil organic carbon decomposition using the DecoChem v1.0 and DecoBio v1.0 models, Geosci. Model Dev., 7, 1519-1533, doi:10.5194/gmd-7-1519-2014, 2014.

Zhu, Q. and Riley, W. J.: Improved modeling of soil nitrogen losses, Nat. Clim. Change, 5, 705-706, 2015.
Zhu, Q. and Zhuang, Q. L.: Parameterization and sensitivity analysis of a process-based terrestrial ecosystem model using adjoint method, J. Adv. Model Earth. Sy., 6, 315-331, 2014.

Zhu, Q., Riley, W. J., Tang, J., and Koven, C. D.: Multiple soil nutrient competition between plants, microbes, and mineral surfaces: model development, parameterization, and example applications in several tropical forests, Biogeosciences Discuss., 12, 40574106, doi:10.5194/bgd-12-4057-2015, 2015a.

Zhu, Q., Riley, W. J., and Tang, J. Y.: A new theory of plant and microbe nutrient competition resolves inconsistencies between observations and Earth System Models, in review, 2015b. 